\title{
IDENTIFIED PARTICLE MEASUREMENTS AT LARGE TRANSVERSE MOMENTA FROM P+P TO AU+AU COLLISIONS AT RHIC.
}

\author{
R. S. HOLLIS* \\ Department of Physics \\ University of Illinois at Chicago \\ 845 W. Taylor Street (MC 273), \\ Chicago, IL 6060\%, USA \\ E-mail:rholli3@uic.edu
}

\begin{abstract}
Measurements of various particle species over an extended momentum range provide a sensitive experimental tool for investigating particle production mechanisms in hadronic collisions. Comparison of the spectral shapes from different collision centralities measured with the STAR detector at RHIC allows one to study the interplay of soft and hard particle production for mesons and investigate various baryon-meson effects. Systematic studies of identified particle spectra for various colliding systems and different incident energies provide additional insights toward the interplay between fragmentation and non-fragmentation contributions to the particle production. In these proceedings we present a systematic study of transverse momentum spectra for charged pions, protons and antiprotons from $\mathrm{Au}+\mathrm{Au}$ and $\mathrm{Cu}+\mathrm{Cu}$ data at $\sqrt{s_{N N}}=200$ and $62.4 \mathrm{GeV}$ as a function of collision centrality. We compare those measurements with $\mathrm{p}+\mathrm{p}$ and $\mathrm{d}+\mathrm{Au}$ data, investigating the system effects on energy loss.
\end{abstract}

\section{Introduction}

Spectral measurements yield valuable information on the state of matter produced in collisions of heavy nuclei in the relativistic energy regime. In these proceedings the importance of identifying particle species from low$\left(\mathrm{p}_{T} \sim 0.4 \mathrm{GeV} / c\right)$ to high- $\mathrm{p}_{T}$ (up to $10 \mathrm{GeV} / c$ ) are discussed in the context of partonic propagation through the hot, dense medium produced in these collisions at the Relativistic Heavy-Ion Collider (RHIC).

Hard partonic interactions, occurring in the early stages of the collision, are known to produce high momentum particles resulting from quark or

*For the STAR Collaboration 
gluon scattering and subsequent fragmentation ${ }^{\mathrm{a}}$. These hard scatterings still occur in heavy-ion collisions, although the partons (through their final products) are found to undergo modification upon propagating the hot, dense medium created during the collision.

High- $\mathrm{p}_{T}$ particles are a valuable probe that could help to understand parton fragmentation and their interactions with the created hot, dense medium. Understanding of modifications to high- $\mathrm{p}_{T}$ particle distributions can lead to qualitative conclusions on the energy loss mechanisms in the medium. As the unmodified (vacuum fragmentation) distribution of high$\mathrm{p}_{T}$ particles is known from elementary $\mathrm{p}+\mathrm{p}$ collision data and is well described by $\mathrm{pQCD}$ calculations, comparative analysis provides a distinct advantage for high- $\mathrm{p}_{T}$ particles.

Various particle species at intermediate- to high- $\mathrm{p}_{T}$ are expected to have very different contributions from quark and gluon jet fragmentation ${ }^{1,2}$. Specifically, intermediate- $\mathrm{p}_{T}$ protons come predominantly from gluon jets, due to their softer fragmentation function. In fragmentation, the majority of pions originate from quark jets by string breaking into quark-antiquark pairs in the intermediate- $\mathrm{p}_{T}$ range. One can then study color-charge differences of energy loss for quark and gluon jets via the identified particle spectra.

It is expected that effects of jet-medium interactions on the final particle distributions are strongly dependent on the size of that medium. Spectral shapes $^{3}$ and azimuthal correlations ${ }^{4}$ in the most central (or fully overlapping) collisions are found to be subjected to the highest modification, whilst $\mathrm{d}+\mathrm{Au}$ or peripheral $\mathrm{Au}+\mathrm{Au}$ collisions are relatively unmodified. The addition of the smaller $\mathrm{Cu}+\mathrm{Cu}$ collision system augments the information in this regard, bridging the gap between $\mathrm{d}+\mathrm{Au}$ and peripheral $\mathrm{Au}+\mathrm{Au}$ collisions.

In these proceedings, the centrality, system size and $\mathrm{p}_{T}$ dependence of the energy loss is explored in the context of the nuclear modification factor and the magnitude of relative baryon enhancement found in such collisions.

\section{Detector and Methods}

The identified particle spectra presented here utilize data collected by the STAR detector at RHIC over the past 6 years. The main apparatus is the time projection chamber, TPC, which covers the full azimuth over the rapidity range $|\eta|<1.8$. The ionization energy loss in the TPC

afrom $\mathrm{p}+\mathrm{p}$ collisions 
is used to identify $\pi^{ \pm}, \mathrm{K}^{ \pm}$and protons and antiprotons in the range $0.3 \mathrm{GeV} / c<\mathrm{p}_{T}<1.2 \mathrm{GeV} / c$, a kinematic region where particles of different masses are clearly separated in $d E / d x$. For the high- $\mathrm{p}_{T}$ regime $\left(\mathrm{p}_{T}>2.5 \mathrm{GeV} / c\right)$, the $\mathrm{p}_{T}$ dependence of the relativistic rise of the ionization energy loss is used to statistically separate the particles. Data from these two $\mathrm{p}_{T}$ regions are augmented by additional coverage afforded by the prototype Time of Flight detector, which identifies particles in the range $0.2 \mathrm{GeV} / c<\mathrm{p}_{T}<3.0 \mathrm{GeV} / c$. More details of the analysis techniques used can be found in Refs. ${ }^{1,5}$.

\section{Results and Discussion}

\subsection{Nuclear Modification Factor}

Modification to the spectral distributions due to the created medium in the heavy-ion collisions are most directly seen in the ratio with an appropriate reference spectra from $\mathrm{p}+\mathrm{p}$ collisions. Such a ratio, scaled by the number of binary collisions $\left(N_{b i n}\right)$, is commonly referred to as the Nuclear Modification Factor (denoted as $\mathrm{R}_{A A}$ ). As can be seen from Fig. 1a, $\pi^{+}+\pi^{-}$ production is significantly suppressed at high- $\mathrm{p}_{T}$ for the most central data compared to the expectation from the binary scaled $\mathrm{p}+\mathrm{p}$ reference. For more peripheral events, the modification is smaller and is found to extrapolate back smoothly to the $\mathrm{p}+\mathrm{p}$ reference (Fig. 1b). These features of the data have been explained by induced energy loss of the partons traversing the hot, dense medium. Such an effect reproduces the centrality dependence of the partonic energy loss found in $\operatorname{data}^{6}$. $\mathrm{Cu}+\mathrm{Cu}$ data add to this systematic study of system size effects of energy loss in heavy ion collisions. A comparison of the same average system size (or number of participants) reveals little difference between the two systems, see Fig. 1.

\subsection{Baryon to meson enhancement}

One of the intriguing observations at RHIC is the increase in the number of baryons relative to mesons in the intermediate- $\mathrm{p}_{T}$ region as compared to more elementary collisions, $\mathrm{p}+\mathrm{p}$ and $e^{+}+e^{-}$. This enhancement, illustrated in Fig. 2a, is seen to depend strongly on the centrality of the collision. With the multiple particle identification techniques, the STAR results completely cover a wide range of transverse-momentum. The relative enhancement, which is found to be maximal at $\mathrm{p}_{T} \sim 2-3 \mathrm{GeV} / c$, is more predominant for the most central $^{2}$ collisions. For peripheral ${ }^{2}$ collisions, the enhancement over $\mathrm{p}+\mathrm{p}^{1}$ collisions is found to diminish leaving the 

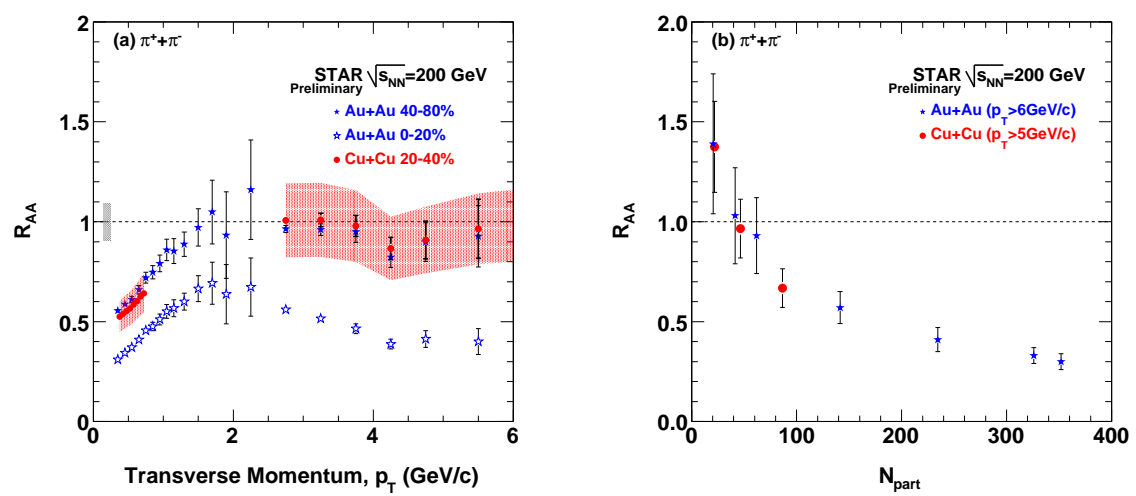

Figure 1. Panel (a) shows the transverse momentum dependence of the nuclear modification factor for $\pi^{+}+\pi^{-}$in $\mathrm{Au}+\mathrm{Au}$ collisions at $\sqrt{s_{N N}}=200 \mathrm{GeV}$ for a central and a peripheral centrality bin. For comparison, $\mathrm{Cu}+\mathrm{Cu}$ data at the same energy is shown for an equivalent $N_{\text {part }}$ bin to the peripheral $\mathrm{Au}+\mathrm{Au}$. The shaded band over the data represents the systematic uncertainty in the $\mathrm{Cu}+\mathrm{Cu}$ data, the grey shaded band illustrates the scale uncertainty from $N_{\text {coll }}$. Panel (b) shows the centrality dependence of the energy loss for the same data, averaged for $\mathrm{p}_{T}>6 \mathrm{GeV} / c$.

data essentially unmodified. $\mathrm{d}+\mathrm{Au} \mathrm{data}^{1}$, at $\sqrt{s_{N N}}=200 \mathrm{GeV}$, is found to exhibit no enhancement relative to the $\mathrm{p}+\mathrm{p}$ collisions. For particles with a higher transverse momenta, the enhancement disappears with the ratio approaching the $\mathrm{p}+\mathrm{p}$ reference at $\mathrm{p}_{T} \sim 5 \mathrm{GeV} / c$.

If indeed protons are predominantly produced in gluon jets and pions in quark jets, two possible explanations of these effects can be discussed. Firstly, one could consider that a gluon jet could be more easily propagated through the medium than a quark jet, leading to an increase in the number of protons in the intermediate- $\mathrm{p}_{T}$ region. This, however, contradicts theoretical predictions ${ }^{6}$ where an opposite effect was expected. Alternatively, more gluon jets could be initially produced, or induced, for the more central data. The latter appears to be the more plausible, as the highest$\mathrm{p}_{T}$ data exhibits little or no enhancement over the $\mathrm{p}+\mathrm{p}$ data, indicating a similar energy loss for gluons and quarks. This is further substantiated by the comparison of pion and proton $R_{c p}$ (Fig. $2 \mathrm{~b}$ ), where no difference is found in the suppression at high- ${ }_{T}{ }^{2}$. Alternative approaches to explain the phenomenon observed in the data, have also been developed. For example, the recombination/fragmentation picture of thermal/shower partons has had success at describing this data ${ }^{8}$. To distinguish between the 

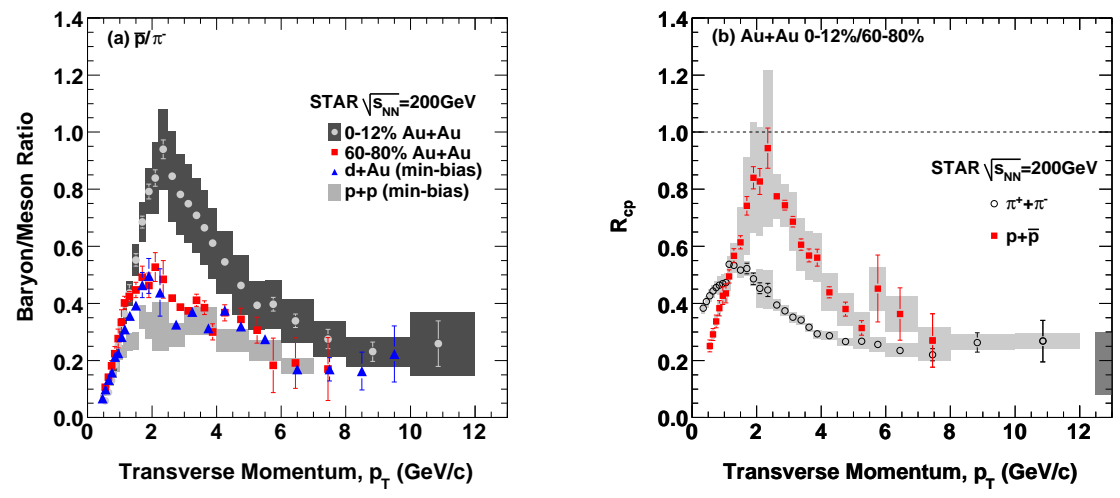

Figure 2. Panel (a) shows the transverse momentum dependence of the baryon-tomeson $\left(\bar{p} / \pi^{-}\right)$ratio in central (0-12\%, circles) and peripheral (60-80\%, squares) $\mathrm{Au}+\mathrm{Au}$ collisions at $\left.\sqrt{s_{N N}}\right)=200 \mathrm{GeV}$. Data from $\mathrm{p}+\mathrm{p}$ (shaded bands) and $\mathrm{d}+\mathrm{Au}$ (triangles) collisions are shown for reference. Panel (b) shows the spectral modification $\left(\mathrm{R}_{C P}\right)$ for pions (open circles) and protons (closed squares) for central (0-12\%) relative to peripheral (60-80\%) collisions. The light shaded bands represent the point to point systematic uncertainty. The darker shaded band represents the normalization systematic uncertainty in the number of binary collisions.

different proposed mechanisms, further differential analysis is needed, perhaps by selecting on the medium path length via the collision's reaction plane-dependent analysis.

Additional information on the observed enhancement of baryons has come from two further sources of systematic study. Firstly, the RHIC facility has produced collisions at a reduced center-of-mass energy of $\sqrt{s_{N N}}=62.4 \mathrm{GeV}$. The measured baryon enhancement is also observed for this incident energy, although the effect is magnified for the proton over pion, presumably due to higher baryon transport for this lower energy. For the antiproton over pion ratio, the enhancement is lower due to a smaller number of primordial anti-particles being produced ${ }^{7}$. A second systematic study has found that, through colliding copper nuclei at the same centerof-mass energy, there is no collision-species dependence of the modification, as long as data with the same number of participants are compared. For both of these, the same systematic $\mathrm{p}_{T}$ dependences are seen: enhancement over $\mathrm{p}+\mathrm{p}$ collisions at intermediate- $\mathrm{p}_{T}$, and no enhancement at high- $\mathrm{p}_{T}$. 


\section{Conclusions}

Measurements of identified protons and pions from low- to high- $\mathrm{p}_{T}$ have proven to be a valuable tool in understanding the particle production and energy loss mechanisms in relativistic heavy-ion collisions. The suppression of pions at high- $\mathrm{p}_{T}$ lead us to conclude that the partons undergo a large energy loss due to a hot, dense medium created during the collisions. Further studies, through the analysis of protons and pions, indicate that the partonic energy loss is similar for both the gluons and quarks. The amount of energy loss suffered by the partons is found to be strongly $N_{\text {part }}$ dependent. For different collision species, the suppression is found to be invariant for the same number of participants.

\section{Acknowledgements}

We thank the RHIC Operations Group and RCF at BNL, and the NERSC Center at LBNL for their support. This work was supported in part by the Offices of NP and HEP within the U.S. DOE Office of Science; the U.S. NSF; the BMBF of Germany; CNRS/IN2P3, RA, RPL, and EMN of France; EPSRC of the United Kingdom; FAPESP of Brazil; the Russian Ministry of Science and Technology; the Ministry of Education and the NNSFC of China; IRP and GA of the Czech Republic, FOM of the Netherlands, DAE,

DST, and CSIR of the Government of India; Swiss NSF; the Polish State Committee for Scientific Research; SRDA of Slovakia, and the Korea Sci. \& Eng. Foundation.

\section{References}

1. J.Adams et al Phys. Lett. B637 (2006) 161

2. B.I.Abelev et al Phys. Rev. Lett. 97 (2006) 152301

3. J.Adams et al Phys. Rev. Lett. 91 (2003) 172302

4. C.Adler et al Phys. Rev. Lett. 90 (2003) 082302

5. J.Adams et al Phys. Lett. B616 (2005) 8

6. I.Vitev Phys. Lett. B639 (2006) 38

7. B.I.Abelev et al arXiv:nucl-ex/0703040 submitted to Phys. Lett. B

8. R.C.Hwa and C.B.Yang Phys.Rev.C 70 (2004) 024905 